\title{
SQUARE INTEGRABLE REPRESENTATIONS OF UNIMODULAR GROUPS
}

\author{
DAVID S. SHUCKER
}

\begin{abstract}
An elementary proof is given showing that if a continuous irreducible unitary representation of a locally compact unimodular group $G$ has one nontrivial square integrable matrix entry, then all its matrix entries are square integrable. This result was first proved by $\mathrm{R}$. Godement.
\end{abstract}

In [2], R. Godement proves that if a continuous irreducible unitary representation of a locally compact unimodular group $G$ has one nontrivial square integrable matrix entry, then all its matrix entries are square integrable. His proof uses the theory of functions of positive type. (See [1].)

In this paper, a simple proof of this result is presented, using only elementary functional analysis and Schur's Lemma.

THEOREM 1 (GODEMENT). Let $G$ be a locally compact, unimodular group, and let $\rho$ be a continuous, irreducible, unitary representation of $G$ on a complex Hilbert space $\mathcal{H}$. Then the following are equivalent:

(a) There exist nonzero vectors $\varphi, \psi \in \mathcal{H}$ such that $\int_{G}|(\rho(h) \varphi, \psi)|^{2}$ dh is finite.

(b) For all $\varphi, \psi \in \mathcal{H}, \int_{G}|(\rho(h) \varphi, \psi)|^{2}$ dh is finite.

(c) The representation $\rho$ is equivalent to a subrepresentation of the regular representation of $G\left(\right.$ on $\left.L^{2}(G)\right)$.

THEOREM 2. If any (hence all) of the conditions in Theorem 1 hold, then there exists a constant $\lambda$ such that for all $\varphi_{1}, \varphi_{2}, \psi_{1}, \psi_{2} \in \mathcal{H}$,

$$
\int_{G}\left(\rho(h) \varphi_{1}, \psi_{1}\right)\left(\rho(h) \varphi_{2}, \psi_{2}\right) d h=\lambda\left(\varphi_{1}, \varphi_{2}\right)\left(\overline{\psi_{1}, \psi_{2}}\right)
$$

(The bar denotes the complex conjugate $\left(\overline{\psi_{1}, \psi_{2}}\right)=\left(\psi_{2}, \psi_{1}\right)$.)

REMARK. In [2], Godement proved Theorem 1 only in the case $\varphi_{0}=\psi_{0}$. His proof is reproduced in [3, Chapter XIV]. A different proof can be found in [4, Chapter I, §5.15].

ProOF OF THEOREM 1.

1. (a) $\Rightarrow$ (b). Assume $0 \neq \varphi_{0}, \psi_{0} \in \mathcal{H}$ and $\int_{G}\left|\left(\rho(h) \varphi_{0}, \psi_{0}\right)\right|^{2} d h<\infty$. Let $D$ be the linear span of the vectors $\rho(k) \varphi_{0}, k \in G$. $\mathscr{D}$ is an invariant subspace of $\mathcal{H}$; that is

Received by the editors December 17, 1982.

1980 Mathematics Subject Classification. Primary 22D10.

(C)1983 American Mathematical Society 0002-9939/83/0000-1290/\$02.00 
$\rho(h)^{\mathscr{D}} \subseteq \mathscr{Q}$ for all $h \in G$. Thus $\mathscr{D}$ is dense in $\mathcal{H}$. By the translation invariance of the measure $d h$,

$$
\begin{aligned}
\int_{G}\left|\left(\rho(h) \rho(k) \varphi_{0}, \psi_{0}\right)\right|^{2} d h & =\int_{G}\left|\left(\rho(h k) \varphi_{0}, \psi_{0}\right)\right|^{2} d h \\
& =\int_{G}\left|\left(\rho(h) \varphi_{0}, \psi_{0}\right)\right|^{2} d h
\end{aligned}
$$

is finite. Thus $\int_{G}\left|\left(\rho(h) \varphi, \psi_{0}\right)\right|^{2} d h$ is finite for all $\varphi \in \mathscr{D}$.

We may therefore define a new inner product $\langle$,$\rangle on \mathscr{D}$ by

$$
\left\langle\varphi_{1}, \varphi_{2}\right\rangle=\left(\varphi_{1}, \varphi_{2}\right)+\int_{G}\left(\rho(h) \varphi_{1}, \psi_{0}\right) \overline{\left(\rho(h) \varphi_{2}, \psi_{0}\right)} d h .
$$

We immediately note the following properties of the new inner product:

(2)

$$
\begin{aligned}
&\langle\varphi, \varphi\rangle \geqslant(\varphi, \varphi) \\
&\left\langle\rho(k) \varphi_{1}, \rho(k) \varphi_{2}\right\rangle=\left(\rho(k) \varphi_{1}, \rho(k) \varphi_{2}\right) \\
&+\int_{G}\left(\rho(h k) \varphi_{1}, \psi_{0}\right) \overline{\left(\rho(h k) \varphi_{2}, \psi_{0}\right)} d h \\
&=\left(\varphi_{1}, \varphi_{2}\right)+\int_{G}\left(\rho(h) \varphi_{1}, \psi_{0}\right) \overline{\left(\rho(h) \varphi_{2}, \psi_{0}\right)} d h=\left\langle\varphi_{1}, \varphi_{2}\right\rangle
\end{aligned}
$$

Let $\mathcal{H}_{1}$ be the completion of $\mathscr{D}$ with respect to the inner product $\langle$,$\rangle . By (3), \rho(k)$ extends uniquely to a unitary operator $\rho_{1}(k)$ on $\rho_{1}$ for each $k \in G$.

Let $T$ be the identity map from $\mathscr{D}$ considered as a subset of $\mathcal{H}_{1}$ to $\mathscr{D}$ considered as a subset of $\mathcal{H}$. From (2), we see $T$ has norm at most 1 . Thus $T$ extends uniquely to a bounded linear operator (which we still denote by $T$ ) from $\mathcal{H}_{1}$ to $\mathcal{H},|T| \leqslant 1$. For $k \in G$, the bounded operators $T \rho_{1}(k)$ and $\rho(k) T$ agree on the dense set $\mathscr{D} \subseteq \mathcal{H}_{1}$, and are thus equal. The adjoint $T^{*}$ of $T$ is a contraction from $\mathcal{H}$ to $\mathcal{H}_{1}$. For $k \in G$, we have

$$
\rho_{1}(k) T^{*}=\left[T \rho_{1}\left(k^{-1}\right)\right]^{*}=\left[\rho\left(k^{-1}\right) T\right] *=T^{*} \rho(k) .
$$

Considering next the bounded operator $T T^{*}$ from $\mathcal{H}$ to itself, for all $k$ in $G$ we have

$$
T T^{*} \rho(k)=T \rho_{1}(k) T^{*}=\rho(k) T T^{*} .
$$

By Schur's Lemma, $T T^{*}$ is some multiple $M$ of the identity.

The range of $T$ includes $\mathscr{D}$ and is therefore dense in $\mathcal{H}$, so $T^{*}$ is injective. Thus $M \neq 0$, for if $T T^{*} \varphi$ is zero, then $\left\|T^{*} \varphi\right\|=\left(T T^{*} \varphi, \varphi\right)=0$, so $\varphi=0$.

Furthermore, $T$ is also injective. For assume $\varphi \in \mathcal{H}_{1}, T \varphi=0$. Then there exists a sequence $\left\{\varphi_{n}\right\}$ in $\mathscr{D}$ converging in $\mathcal{H}_{1}$ to $\varphi$. It follows that $\left\{\varphi_{n}\right\}=\left\{T \varphi_{n}\right\}$ converges in $\mathcal{H}$ to $T \varphi=0$, and that the functions $\left(\rho(h) \varphi_{n}, \psi_{0}\right)=\left(\varphi_{n}, \rho\left(h^{-1}\right) \psi_{0}\right)$ form a Cauchy sequence in $L^{2}(G)$. By passing to a subsequence, we may assume $\left(\varphi_{n}, \rho\left(h^{-1}\right) \psi_{0}\right)$ converges almost everywhere in $G$ to its $L^{2}$ limit. But $\left(\varphi_{n}, \rho\left(h^{-1}\right) \psi_{0}\right)$ converges uniformly to zero. Thus $\left(\varphi_{n}, \rho\left(h^{-1}\right) \psi_{0}\right)$ converges in $L^{2}(G)$ to zero, and $\left\{\varphi_{n}\right\}$ converges to $\mathcal{H}_{1}$ to 0 .

For $\varphi \in \mathscr{D} \subseteq \mathcal{H}$, we have $T T^{*} \varphi=M \varphi=T(M \varphi)$ and hence, because $T$ is injective, we have $T^{*} \varphi=M \varphi$. Now for $\varphi \in \mathscr{D} \subseteq \mathcal{H}_{1}$, we have $T^{*} T \varphi=T^{*} \varphi=M \varphi$; by continuity, this holds for all $\varphi \in \mathcal{H}_{1}$. 
For $\varphi \in \mathscr{D}$, we have

$$
M\langle\varphi, \varphi\rangle=\left\langle T^{*} T \varphi, \varphi\right\rangle=(T \varphi, T \varphi)=(\varphi, \varphi),
$$

which we may rewrite, using (1), as

$$
M(\varphi, \varphi)+M \int_{G}\left|\left(\rho(h) \varphi, \psi_{0}\right)\right|^{2} d h=(\varphi, \varphi) .
$$

Substituting $\varphi_{0}$ for $\varphi$, we see $0<M<1$, for if $M=1$, then the dense set $\mathscr{D}$ is orthogonal to the nonzero vector $\psi_{0}$.

We have thus established, for $\varphi \in \mathscr{Q}$, the equality

$$
\int_{G}\left|\left(\rho(h) \varphi, \psi_{0}\right)\right|^{2} d h=\frac{1-M}{M}(\varphi, \varphi)<\infty .
$$

Now consider an arbitrary vector $\varphi \in \mathcal{H}$. Let $\left\{\varphi_{n}\right\}$ be a sequence in $\mathscr{D}$ that converges in $\mathcal{H}$ to $\varphi$. By (4), we see that the sequence of functions $\left(\rho(h) \varphi_{n}, \psi_{0}\right)$ is Cauchy in $L^{2}(G)$ and converges in $L^{2}(G)$ to a function with norm

$$
\lim _{n \rightarrow \infty}\left[\frac{1-M}{M}\left(\varphi_{n}, \varphi_{n}\right)\right]^{1 / 2}=\left[\frac{1-M}{M}(\varphi, \varphi)\right]^{1 / 2} .
$$

By passing to a subsequence, we may assume $\left(\rho(h) \varphi_{n}, \psi_{0}\right)=\left(\varphi_{n}, \rho\left(h^{-1}\right) \psi_{0}\right)$ converges almost everywhere to its $L^{2}$ limit. But $\left(\varphi_{n}, \rho\left(h^{-1}\right) \psi_{0}\right)$ converges uniformly to $\left(\varphi, \rho\left(h^{-1}\right) \psi_{0}\right)$. We have now established (4) for all $\varphi \in \mathcal{H}$.

We have so far proved that if $0 \neq \varphi_{0}, \psi_{0} \in \mathcal{K}$ and $\int_{G}\left|\left(\rho(h) \varphi_{0}, \psi_{0}\right)\right|^{2} d h$ is finite, then there exists a constant $M_{\psi_{0}}$ such that $\int_{G}\left|\left(\rho(h) \varphi, \psi_{0}\right)\right|^{2} d h=M_{\psi_{0}}(\varphi, \varphi)$ is finite for all $\varphi \in \mathcal{H}$.

As

$$
\begin{aligned}
\int_{G}|(\rho(h) \varphi, \psi)|^{2} d h & =\int_{G}\left|\left(\varphi, \rho\left(h^{-1}\right) \psi\right)\right|^{2} d h \\
& =\int_{G}|(\varphi, \rho(h) \psi)|^{2} d h=\int_{G}|(\rho(h) \psi, \varphi)|^{2} d h,
\end{aligned}
$$

it also follows under the above hypotheses that

$$
\int_{G}\left|\left(\rho(h) \varphi_{0}, \psi\right)\right|^{2} d h=M_{\varphi_{0}}(\psi, \psi)
$$

is finite for all $\psi \in \mathcal{H}$. It now follows that $\int_{G}|(\rho(h) \varphi, \psi)|^{2} d h$ is finite for all $\varphi, \psi \in \mathcal{H}$.

2. (b) $\Rightarrow$ (c). The map $A_{\varphi}: \mathcal{H} \rightarrow L^{2}(G)$ defined by $\left(A_{\varphi} \psi\right)(h)=(\psi, \rho(h) \varphi)$ is easily seen to be an intertwining operator between $\rho$ and the left regular representation $\pi$ : $[\pi(g) f](h)=f\left(g^{-1} h\right)$ on $L^{2}(G)$.

$$
\begin{aligned}
{\left[\left(\pi(g) \circ A_{\varphi}\right) \psi\right](h) } & =\left[\pi(g)\left(A_{\varphi} \psi\right)\right](h)=\left(A_{\varphi} \psi\right)\left(g^{-1} h\right) \\
& =\left(\psi, \rho\left(g^{-1} h\right) \varphi\right)=(\rho(g) \psi, \rho(h) \varphi)=\left[A_{\varphi}(\rho(g) \psi)\right](h) \\
& =\left[\left(A_{\varphi} \circ \rho(g)\right) \psi\right](h) .
\end{aligned}
$$

Furthermore, by Theorem 2 (whose proof below requires only condition (b) of Theorem 1), $A_{\varphi}$ is bounded. In fact, if $\varphi$ is chosen to have norm $\lambda^{-1 / 2}$, then $A_{\varphi}$ is an isometry. 
3. (c) $\Rightarrow$ (a). The reader is referred to $[4, \S 5.15]$ for a simple proof of this fact.

Proof of TheOREM 2. By the Schwarz inequality, the integral

$$
\int_{G}\left(\rho(h) \varphi_{1}, \psi_{1}\right) \overline{\left(\rho(h) \varphi_{2}, \psi_{2}\right)} d h
$$

is well defined and bounded in absolute value by $M_{\psi_{1}}^{1 / 2} M_{\psi_{2}}^{1 / 2}\left\|\varphi_{1}\right\|\left\|\varphi_{2}\right\|$ for all $\varphi_{1}$, $\varphi_{2}, \psi_{1}, \psi_{2} \in \mathcal{H}$. For fixed $\psi_{1}$ and $\psi_{2}$, the integral is a bounded sesquilinear form on $\mathcal{H}$. There is therefore a bounded operator $T_{\psi_{1}, \psi_{2}}$ on $\mathcal{H},\left\|T_{\psi_{1}, \psi_{2}}\right\| \leqslant M_{\psi_{1}}^{1 / 2} M_{\psi_{2}}^{1 / 2}$, such that the integral equals $\left(T_{\psi_{1}, \psi_{2}} \varphi_{1}, \varphi_{2}\right)$ for all $\varphi_{1}, \varphi_{2} \in \mathcal{H}$.

From the translation invariance of the measure $d h$, we have for all $k \in G, \varphi_{1}$, $\varphi_{2} \in \mathcal{H}$

$$
\begin{aligned}
\left(T_{\psi_{1}, \psi_{2}} \varphi_{1}, \varphi_{2}\right) & =\left(T_{\psi_{1}, \psi_{2}} \rho(k) \varphi_{1}, \rho(k) \varphi_{2}\right) \\
& =\left(\rho(k)^{-1} T_{\psi_{1}, \psi_{2}} \rho(k) \varphi_{1}, \varphi_{2}\right) .
\end{aligned}
$$

By Schur's Lemma, $T_{\psi_{1}, \psi_{2}}$ is some multiple $M_{\psi_{1}, \psi_{2}}$ of the identity

$$
\int_{G}\left(\rho(h) \varphi_{1}, \psi_{1}\right) \overline{\left(\rho(h) \varphi_{2}, \psi_{2}\right)} d h=M_{\psi_{1}, \psi_{2}}\left(\varphi_{1}, \varphi_{2}\right)
$$

and

$$
\begin{aligned}
\int_{G}\left(\rho(h) \varphi_{1}, \psi_{1}\right) \overline{\left(\rho(h) \varphi_{2}, \psi_{2}\right)} d h & =\int_{G}\left(\varphi_{1}, \rho\left(h^{-1}\right) \psi_{1}\right) \overline{\left(\varphi_{2}, \rho\left(h^{-1}\right) \psi_{2}\right)} d h \\
& =\int_{G}\left(\varphi_{1}, \rho(h) \psi_{1}\right) \overline{\left(\varphi_{2}, \rho(h) \psi_{2}\right)} d h \\
& =\int_{G}\left(\rho(h) \psi_{2}, \varphi_{2}\right) \overline{\left(\rho(h) \psi_{1}, \varphi_{1}\right)} d h \\
& =M_{\varphi_{2}, \varphi_{2}}\left(\psi_{2}, \psi_{1}\right) .
\end{aligned}
$$

Taking $\varphi_{1}=\varphi_{2}=e=$ a unit vector in (5) and (6), we find $M_{\psi_{1}, \psi_{2}}=M_{e, e}\left(\psi_{2}, \psi_{1}\right)$.

$$
\begin{aligned}
\int_{G}\left(\rho(h) \varphi_{1}, \psi_{1}\right) \overline{\left(\rho(h) \varphi_{2}, \psi_{2}\right)} d h & =M_{\psi_{1}, \psi_{2}}\left(\varphi_{1}, \varphi_{2}\right)=M_{e, e}\left(\psi_{2}, \psi_{1}\right)\left(\varphi_{1}, \varphi_{2}\right) \\
& =M_{e, e}\left(\varphi_{1}, \varphi_{2}\right) \overline{\left(\psi_{1}, \psi_{2}\right)} .
\end{aligned}
$$

ACKnowledgment. The author is grateful to Ray Kunze and David Gurarie for valuable discussions. The author thanks the referee for references [ 3 and 4 ], and for pointing out the equivalence of condition (c) of Theorem 1 with square integrability.

\section{REFERENCES}

1. R. Godement, Sur les relations d'orthgonalité de V. Bargmann. I. Résultats préliminaires, C. R. Acad. Sci. Paris Sér. A-B 225 (1947), 521.

2. __ Sur les relations d'orthogonalité de V. Bargmann. II. Démonstration générale, C. R. Acad. Sci. Paris Sér. A-B 225 (1947), 657.

3. J. Dixmier, C*-algebras, North-Holland, New York, 1977.

4. A. Borel, Représentations de groupes localement compacts, Lecture Notes in Math., Springer-Verlag, New York, 1972 .

Department of Mathematics, Texas A \& M University, College Station, Texas 77843 